\title{
ON EIGENVALUE PROBLEMS OF THE $p$-LAPLACIAN WITH NEUMANN BOUNDARY CONDITIONS
}

\author{
YIN XI HUANG
}

(Communicated by Barbara L. Keyfitz)

\begin{abstract}
We study the nonlinear eigenvalue problem

$$
-\Delta_{p} u=\lambda m(x)|u|^{p-2} u \quad \text { in } \Omega, \quad \frac{\partial u}{\partial n}=0 \quad \text { on } \partial \Omega, \text { where } p>1, \lambda \in \mathbf{R} .
$$

For $\int_{\Omega} m(x)<0$, we prove that the first positive eigenvalue $\lambda_{1}$ exists and is simple and unique, in the sense that it is the only eigenvalue with a positive eigenfunction. In the case $\int_{\Omega} m(x)=0$, we prove that $\lambda_{0}=0$ is the only eigenvalue with a positive eigenfunction.
\end{abstract}

\section{INTRODUCTION}

Let $\Omega$ be a bounded domain in $R^{n}$ with smooth boundary $\partial \Omega$. For a given number $p>1$ and a function $m(x) \in C(\Omega)$, we consider the following nonlinear eigenvalue problem with Neumann boundary condition

$$
\begin{aligned}
-\Delta_{p} u & =\lambda m(x)|u|^{p-2} u & & \text { in } \Omega \\
\frac{\partial u}{\partial n} & =0 & & \text { on } \partial \Omega
\end{aligned}
$$

where $\Delta_{p} u=\operatorname{div}\left(|\nabla u|^{p-2} \nabla u\right)$ is the $p$-Laplacian and $\lambda \in \mathbf{R}$.

The operator $-\Delta_{p}$ with $p \neq 2$ arises from a variety of physical phenomena. It is used in non-Newtonian fluids, in some reaction-diffusion problems, as well as in flow through porous media. It also appears in nonlinear elasticity, glaceology, and petroleum extraction. Diaz [4] collected detailed references on physical background and presented mathematical treatments of free boundary problem associated with $-\Delta_{p}$. Recently, the eigenvalue problems of $-\Delta_{p}$ with indefinite weight with respect to Dirichlet boundary conditions were investigated by Otani and Teshima [9] and Anane [1], while Guedda and Veron [5] studied the one-dimensional bifurcation phenomena of $-\Delta_{p}$. We refer to $[1,4,5,9]$

Received by the editors March 10, 1989 and, in revised form, July 17, 1989.

1980 Mathematics Subject Classification (1985 Revision). Primary 35P30; Secondary 35J60.

Key words and phrases. p-Laplacian, eigenvalue, Neumann boundary condition.

This work was partly supported by the Izaak Walton Killam Memorial Scholarship at the University of Alberta. 
for more references and for other aspects of $-\Delta_{p}$. In the case $p=2$, eigenvalue problems of second-order elliptic operator with indefinite weight were studied by many authors. Senn and Hess [10], Hess and Senn [7] studied eigenvalue problems with Neumann boundary conditions. Bandle, Pozio, and Tesei [2] studied the existence and uniqueness of positive solutions to some nonlinear Neumann problems. The purpose of this paper is to study the existence of a positive real number $\lambda_{1}$, the first eigenvalue, such that (1)-(2) has a positive solution $u_{1}$ if and only if $\lambda=\lambda_{1}$ and such that $\lambda_{1}$ is simple in the sense that such solution $u_{1}$ is unique up to a constant multiplication. Our approach in this paper follows the technique of [10]. However, since the problem we study is nonlinear and the associated operator is not selfadjoint, the Krein-Rutman theory used extensively in [10] is not applicable here.

The rest of the paper is organized as follows. In $\S 2$, we introduce some known results for the $p$-Laplacian and set up the framework for later sections. In $\S 3$, the existence, uniqueness, and simplicity of the first positive eigenvalue $\lambda_{1}$ are proved for the case $\int_{\Omega} m(x) d x<0$. The exceptional case $\int_{\Omega} m(x) d x=0$ is discussed in $\S 4$, where we prove that $\lambda_{0}=0$ is the only eigenvalue with a corresponding positive eigenfunction.

\section{Preliminaries}

In this section, we will set up an appropriate functional analysis framework for our problem. We first state some fundamental properties of the solutions of the following Neumann boundary value problem,

$$
\begin{gathered}
-\Delta_{p} u+\varepsilon|u|^{p-2} u=f \text { in } \Omega \\
\frac{\partial u}{\partial n}=0 \text { on } \partial \Omega,
\end{gathered}
$$

where $\Omega \subset \mathbf{R}^{n}$ is a bounded domain with smooth boundary, $p>1$ and $\varepsilon>0$.

By Theorem 2.1 of Lions [8, p. 171], and Theorem 2.3 of [8, p. 176], (3)(4) has a unique solution $u \in W^{1, p}(\Omega)$ for any given $f \in L^{q}(\Omega)$ where $q$ is such that $\frac{1}{p}+\frac{1}{q}=1$. Regularity theorems (cf. Tolksdorf [11, p. 806] or Di Benedetto [3]), further show that if $f \in L^{\infty}(\Omega)$, then $u \in C^{1+\alpha}(\bar{\Omega})$ for some $0<\alpha<1$.

Let $J u:=|u|^{p-2} u$, and

$$
\mathscr{D} \equiv \mathscr{D}(L)=\left\{u \in L^{\infty}(\Omega) \mid u \text { solves (3)-(4) for some } f \in L^{\infty}(\Omega)\right\} .
$$

On $\mathscr{D}$ define the operator $L$ by $L u=-\varepsilon J u+f$, where $f \in L^{\infty}(\Omega)$ is such that $u$ solves (3)-(4) for this $f$.

Then for any $\varepsilon>0, L+\varepsilon J$ is injective and maps $\mathscr{D}$ onto $L^{\infty}(\Omega)$. Thus its inverse $R_{\varepsilon}=(L+\varepsilon J)^{-1}: L^{\infty}(\Omega) \rightarrow \mathscr{D} \subset L^{\infty}(\Omega)$ exists. Since $C^{1+\alpha}(\bar{\Omega})$ is embedded in $L^{\infty}(\Omega)$ compactly and densely, $R_{\varepsilon}: L^{\infty}(\Omega) \rightarrow L^{\infty}(\Omega)$ is compact. We now show that $R_{\varepsilon}$ is strictly positive, i.e. for $f \geq 0$ and $f \not \equiv 0$, $R_{\varepsilon} f>0$. Indeed, let $u=R_{\varepsilon} f$, then $-\Delta_{p} u+\varepsilon|u|^{p-2} u=f$. Let $\tilde{u}=\min (u, 0)$. 
Multiplying the previous equation by $\tilde{u}$ on both sides and integrating, we get $u \geq 0$. Lemma 4 of Otani and Teshima [9] further shows that $u>0$ on $\bar{\Omega}$. Thus $R_{\varepsilon}$ is strictly positive.

Let $M: L^{\infty}(\Omega) \rightarrow L^{\infty}(\Omega)$ denote the multiplication operator induced by the weight function $m(x) \in C(\Omega)$, i.e. $M u=m(x) u$. Then the eigenvalue problem (1)-(2) is equivalent to the following operator equation,

$$
L u=\lambda M J u,
$$

in $L^{\infty}(\Omega)$, for $\lambda \in \mathbf{R}$.

A simple integration shows that if $m(x)$ is of constant sign, there is no nonzero eigenvalue $\lambda$ of $(1)-(2)$ with a positive eigenfunction. Thus from now on we always assume that $m(x)$ changes sign in $\Omega$. Also, without loss of generality, we assume that $\|m(x)\|_{\infty}<1$.

For $\alpha>0$, define

$$
K_{\alpha}=R_{\alpha}(M+1) J .
$$

Then $K_{\alpha}$ is compact and strictly positive. We note that $u$ is a solution of (5) if and only if $u$ is a solution of

$$
u=\lambda^{\frac{1}{p-1}} K_{\lambda} u \text {. }
$$

\section{Main Results}

We start with the construction of a subsolution to (6). The same technique was employed in $[2,6,10]$ for the same purpose.

Lemma 1. There exist $\alpha_{0}>0$ and $w_{0} \in L^{\infty}(\Omega), w_{0} \geq 0$, such that

$$
w_{0} \leq \alpha_{0}^{\frac{1}{p-1}} K_{\alpha_{0}} w_{0}
$$

i.e., $w_{0}$ is a subsolution to (6) for $\lambda=\alpha_{0}$.

Proof. Let $\Omega^{+}=\{x \in \Omega \mid m(x)>0\}$ and let $U \subset \Omega^{+}$be an open connected domain. Set $\delta=\min _{x \in U} m(x)$. It is well known that there exist $\gamma_{1}>0$, and $\tilde{w}_{1} \in L^{\infty}(U), \tilde{w}_{1}>0$, such that

$$
-\Delta_{p} \tilde{w}_{1}=\gamma_{1} J \tilde{w}_{1} \quad \text { in } U, \quad \tilde{w}_{1}=0 \text { on } \partial U
$$

Set $\alpha_{0}=\gamma_{1} / \delta$, then

$$
\left(-\Delta_{p}+\alpha_{0} J\right) \tilde{w}_{1}=\alpha_{0}(\delta+1) J \tilde{w}_{1} .
$$

Now let

$$
w_{0}= \begin{cases}\tilde{w}_{1} & \text { in } U \\ 0 & \text { in } \bar{\Omega} \backslash U .\end{cases}
$$

We claim that

$$
w_{0} \leq \alpha_{0}^{\frac{1}{p-1}}\left(L+\alpha_{0} J\right)^{-1}(\delta+1) J w_{0} .
$$


Indeed, let $v=\alpha_{0}^{\frac{1}{p-1}}\left(L+\alpha_{0} J\right)^{-1}(\delta+1) J w_{0}$. Since $(\delta+1) J w_{0} \geq 0, v>0$ on $\bar{\Omega}$. Thus $v>w_{0}$ in $\bar{\Omega} \backslash U$.

Since $w_{0}$ and $v$ solve

$$
\begin{aligned}
\left(-\Delta_{p}+\alpha_{0} J\right) w_{0} & =\alpha_{0}(\delta+1) J w_{0} \text { in } \Omega, & & w_{0}=0 \text { on } \partial U \\
\left(-\Delta_{p}+\alpha_{0} J\right) v & =\alpha_{0}(\delta+1) J w_{0} \text { in } \Omega, & & v>0 \text { on } \partial U
\end{aligned}
$$

respectively, by maximum principle (cf. Lemma 3 of [9], or Proposition 3.2.1 of [11]), we conclude that $v \geq w_{0}$ in $U$. Thus (8) follows.

Since $(\delta+1) J w_{0} \leq(M+1) J w_{0},(8)$ and maximum principle imply that $w_{0} \leq \alpha_{0}^{\frac{1}{p-1}} K_{\alpha_{0}} w_{0}$. This completes the proof of the lemma.

Lemma 2. For this $\alpha_{0}$, there exist $\alpha_{1}$ such that $0<\alpha_{1} \leq \alpha_{0}$ and $w_{1} \in L^{\infty}(\Omega)$, $w_{1}>0$, such that

$$
w_{1}=\alpha_{1}^{\frac{1}{p-1}} K_{\alpha_{0}} w_{1}=\alpha_{1}^{\frac{1}{p-1}}\left(L+\alpha_{0} J\right)^{-1}(M+1) J w_{1} .
$$

Proof. Consider the following eigenvalue problem

$$
\begin{aligned}
-\Delta_{p} u+\alpha_{0}|u|^{p-2} u & =\lambda(m(x)+1)|u|^{p-2} u, \quad \text { in } \Omega \\
\frac{\partial u}{\partial n} & =0, \quad \text { on } \partial \Omega .
\end{aligned}
$$

Then the arguments in the proof of Theorem 1 in [9] yield the existence of the first eigenvalue $\tilde{\lambda}_{1}>0$ with a positive eigenfunction $w_{1}>0$. It is easy to see that $w_{1}$ satisfies (9) with $\alpha_{1}=\tilde{\lambda}_{1}$. Since $\tilde{\lambda}_{1}$ can be expressed as

$$
\tilde{\lambda}_{1}=\inf _{u \in W^{1 . p}} \frac{\int|\nabla u|^{p}+\alpha_{0} \int|u|^{p}}{\int(m+1)|u|^{p}}
$$

we conclude that $\alpha_{0} \geq \tilde{\lambda}_{1}=\alpha_{1}$. This completes the proof of the lemma.

Now, since $w_{1}$ satisfies (9),

$$
\left(L+\alpha_{0} J\right) w_{1}=\alpha_{1}(M+1) J w_{1} .
$$

Thus

$$
\left(L+\alpha_{1} J\right) w_{1}=\alpha_{1}(M+1) J w_{1}-\left(\alpha_{0}-\alpha_{1}\right) J w_{1} .
$$

Hence we conclude that

$$
w_{1} \leq \alpha_{1}^{\frac{1}{p-1}} K_{\alpha_{1}} w_{1},
$$

since $\left(\alpha_{0}-\alpha_{1}\right) \geq 0$ and $\left(L+\alpha_{1} J\right)^{-1}$ is strictly positive.

Repeated application of Lemma 2 yields a decreasing sequence of real numbers $\alpha_{n} \geq 0$ and a sequence of functions $w_{n} \in L^{\infty}(\Omega), w_{n} \geq 0$, such that

$$
\left(L+\alpha_{n} J\right) w_{n}=\alpha_{n}(M+1) w_{n}-\left(\alpha_{n-1}-\alpha_{n}\right) J w_{n} .
$$

We will normalize $w_{n}$ so that $\left\|w_{n}\right\|_{\infty}=1$. 
Lemma 3. Assume that

$$
\int_{\Omega} m(x) d x<0
$$

Then there exist $\lambda_{1}$ with $0<\lambda_{1} \leq \alpha_{0}$, and $u>0$, such that $L u=\lambda_{1} M J u$.

Proof. From the above we know that $\alpha_{n} \rightarrow \lambda_{1} \in\left[0, \alpha_{0}\right]$. If $\lambda_{1}=0$, then since

$$
L w_{n}=\alpha_{n}(M+1) J w_{n}-\alpha_{n-1} J w_{n},
$$

we obtain that $\left\|L w_{n}\right\|_{\infty} \leq 3 \alpha_{n-1}\left\|w_{n}\right\|_{\infty}=3 \alpha_{n-1}$. Hence $L w_{n} \rightarrow 0$ in $L^{\infty}$, i.e. $\left\|(L+J) w_{n}\right\|_{\infty} \leq$ const. The compactness of $(L+J)^{-1}$ implies that $w_{n} \rightarrow u$ for some $u \in L^{\infty}(\Omega)$, and $u>0,\|u\|_{\infty}=1$. We further have $L u=0$, and thus $u=1$. Since

$$
0 \leq \int\left|\nabla w_{n}\right|^{p}=\int \alpha_{n} m\left|w_{n}\right|^{p}+\left(\alpha_{n}-\alpha_{n-1}\right) \int\left|w_{n}\right|^{p}
$$

we conclude that

$$
\alpha_{n} \int m\left|w_{n}\right|^{p}>0
$$

This implies that $\int_{\Omega} m(x) d x>0$, which is a contradiction. Hence $\lambda_{1}>0$. Then $\left(L+\alpha_{n} J\right)^{-1} \rightarrow\left(L+\lambda_{1} J\right)^{-1}$ and $K_{\alpha_{n}} \rightarrow K_{\lambda_{1}}$. Thus we have

$$
w_{n}-\alpha_{n}^{\frac{1}{p-1}} K_{\alpha_{n}} w_{n} \rightarrow 0
$$

Since $K_{\alpha_{n}}$ is compact, $\left\{w_{n}\right\}$ lies in a compact subset of $L^{\infty}(\Omega)$. Therefore by going to subsequence, if necessary, we can assume that $w_{n} \rightarrow u$ in $L^{\infty}$ and $u>0$. From (12) we conclude that

$$
u=\lambda_{1}^{\frac{1}{p-1}} K_{\lambda_{1}} u, \quad \text { i.e. } L u=\lambda_{1} M J u \text {. }
$$

This ends the proof of the lemma.

Lemma 4. $\lambda_{1}$ is simple and unique.

Proof. Set

$$
I(u, v)=\int\left(-\Delta_{p} u\right) \cdot \frac{u^{p}-v^{p}}{u^{p-1}}-\int\left(-\Delta_{p} v\right) \cdot \frac{u^{p}-v^{p}}{v^{p-1}}
$$

for $u, v \in W^{1, p}$ and $u, v>0$. Then Proposition 1 of Anane [1] ensures that $I(u, v) \geq 0$, and $I(u, v)=0$ if and only if $u=c v$ for some constant $c$.

If $u_{1}, u_{2}$ are two normalized positive eigenfunctions (i.e. $\left\|u_{i}\right\|_{\infty}=1, i=$ $1,2)$ associated with $\lambda_{1}$, then

$$
\begin{aligned}
I\left(u_{1}, u_{2}\right) & =\int \lambda_{1} m \cdot\left(u_{1}^{p}-u_{2}^{p}\right)-\int \lambda_{1} m \cdot\left(u_{1}^{p}-u_{2}^{p}\right) \\
& =0 .
\end{aligned}
$$

Thus $u_{1} \equiv u_{2}$, and $\lambda_{1}$ is simple. Similarly one can prove that $\lambda_{1}$ is unique. Lemma 4 is proved. 
Now, summarizing the above we have

Theorem 1. Assume that $\int_{\Omega} m<0$. Then the eigenvalue problem (1)-(2) admits a positive eigenvalue $\lambda_{1}$ with a positive eigenfunction; and such $a \lambda_{1}$ is unique and simple.

\section{The eXCEPTiOnal CASE}

In this section we consider the exceptional case

$$
\int_{\Omega} m(x) d x=0 .
$$

Obviously, $\lambda_{0}=0$ is an eigenvalue with $u_{0}=1$ as the eigenfunction to (1)-(2). Furthermore, we have the following

Theorem 2. Assume that $\int_{\Omega} m(x) d x=0$. Then $\lambda_{0}=0$ is the only eigenvalue of (1)-(2) having a positive eigenfunction.

Proof. Suppose not, i.e. assume that there exist a $\lambda_{1} \neq 0$ and $u_{1}>0$ satisfying

$$
-\Delta_{p} u_{1}=\lambda_{1} m u_{1}^{p-1} \text { in } \Omega \text { and } \frac{\partial u_{1}}{\partial u}=0 \text { on } \partial \Omega \text {. }
$$

For any $\delta>0$, let

$$
h\left(u_{1}\right)=\int_{\delta}^{u_{1}} \frac{d t}{t}
$$

Then

$$
\begin{gathered}
\nabla h\left(u_{1}\right)=\frac{1}{u_{1}} \nabla u_{1}, \\
\frac{\partial h\left(u_{1}\right)}{\partial n}=\frac{1}{u_{1}} \frac{\partial u_{1}}{\partial n}=0 \text { on } \partial \Omega
\end{gathered}
$$

and

$$
\begin{aligned}
-\Delta_{p} h\left(u_{1}\right) & =-\nabla\left(\frac{1}{u_{1}^{p-1}}\left|\nabla u_{1}\right|^{p-2} \nabla u_{1}\right) \\
& =-u_{1}^{-(p-1)} \Delta_{p} u_{1}+(p-1) u_{1}^{-p}\left|\nabla u_{1}\right|^{p} \\
& =\lambda_{1} m+(p-1) u_{1}^{-p}\left|\nabla u_{1}\right|^{p} .
\end{aligned}
$$

Integrating (15) yields

$$
-\int \lambda_{1} m=\int(p-1) u_{1}^{-p}\left|\nabla u_{1}\right|^{p}
$$

Combining with (14) we conclude that $\left|\nabla u_{1}\right|=0$, i.e. $u_{1}$ is a constant. Thus from (1)-(2) we conclude that $\lambda_{1}=0$, which is a contradiction. Hence the theorem is proved. 
By the same technique, we have the following more general

Corollary 1. Assume that $u$ is a positive solution to

$$
\begin{gathered}
-\Delta_{p} u=m(x) f(u) \quad \text { in } \Omega \\
\frac{\partial u}{\partial n}=0 \quad \text { on } \partial \Omega
\end{gathered}
$$

where $f^{\prime}(s)>0$ for $s>0, f(s)>0$ for $s>0$. Then $\int_{\Omega} m(x) \leq 0$.

Proof. Let

$$
h(u)=\int_{\delta}^{u} \frac{d t}{[f(t)]^{1 / p-1}},
$$

where $\delta>0$ is fixed. Then $h(u)$ satisfies

$$
\begin{gathered}
-\Delta_{p} h(u)=m(x)+\frac{f^{\prime}(u)}{f^{2}(u)}|\nabla u|^{p} \quad \text { in } \Omega \\
\frac{\partial h(u)}{\partial n}=0 \quad \text { on } \partial \Omega .
\end{gathered}
$$

Integration yields

$$
-\int_{\Omega} m(x)=\int_{\Omega} \frac{f^{\prime}(u)}{f^{2}(u)}|\nabla u|^{p} \geq 0,
$$

and hence the result.

Remark. We note that this corollary holds for nonnegative solutions: we need only consider the integration over the support of $u$ in this case. A direct consequence of this corollary is that if $\int_{\Omega} m(x)>0,(16)-(17)$ has no positive solutions.

\section{ACKNOWLEDGMENTS}

The author is indebted to his supervisor Dr. W. Allegretto for his excellent academic guidance, encouragement, and support and to Dr. Joseph So for helpful discussions during the preparation of this work.

\section{REFERENCES}

1. A. Anane, Simplicité et isolation de la première valeur propre du p-laplacien avec poids, C. R. Acad. Sci. Paris Sér. I Math. 305, (1987), 725-728

2. C. Bandle, M. A. Pozio, and A. Tesei, Existence and uniqueness of solutions of nonlinear Neumann problems, Math. Z. 199, (1988), 257-278

3. E. Di Benedetto, $C^{1+\infty}$ local regularity of weak solutions of degenerate elliptic equations, Nonlinear Anal. 7, (1983), 827-850

4. J. I. Diaz, Nonlinear partial differential equations and free boundaries vol. 1 Elliptic equations, Res. Notes in Math., no. 106, Pitman, London, 1985.

5. M. Guedda and L. Veron, Bifurcation phenomena associated to the p-Laplace operator, Trans. Amer. Math. Soc. 310, (1988), 419-431.

6. P. Hess and T. Kato, On some linear and nonlinear eigenvalue problems with an indefinite weight function, Comm. Partial Differential Equations 5, (1980), 999-1030. 
7. P. Hess and S. Senn, Another approach to elliptic eigenvalue problems with respect to indefinite weight functions, Proc. Conference on Nonlinear Analysis and Optimization, Lecture Notes in Math., vol. 1107, Springer-Verlag, New York, 1984, pp. 106-114.

8. J. L. Lions, Quelques méthodes de résolution des problèmes aux limites non linéaires, Dunod, Paris, 1969.

9. M. Otani and T. Teshima, On the first eigenvalue of some quasilinear elliptic equations, Proc. Japan Acad. Ser. A. Math. Sci. 64, (1988), 8-10.

10. S. Senn and P. Hess, On positive solutions of a linear elliptic eigenvalue problem with Neumann boundary conditions, Math. Ann. 258, (1982), 459-470.

11. P. Tolksdorf, On the Dirichlet problem for quasilinear equations in domains with conical boundary points, Comm. Partial Differential Equations 8, (1983), 773-817.

Department of Mathematics, University of Alberta, Edmonton, Alberta T6G 2 G1 CANADA

Current address: Department of Mathematics and Statistics, University of Calgary, Calgary, Alberta T2N 1N4 Canada 